\title{
Research Square \\ Ferroelectric photosensor network: an advanced hardware solution to real-time machine vision
}

Boyuan Cui

South China Normal University

Zhen Fan ( $\nabla$ fanzhen@m.scnu.edu.cn )

South China Normal University https://orcid.org/0000-0002-1756-641X

Wenjie Li

South China Normal University

Yihong Chen

South China Normal University

Shuai Dong

South China Normal University

Zhengwei Tan

South China Normal University

Shengliang Cheng

South China Normal University

Bobo Tian

East China Normal University https://orcid.org/0000-0002-4251-2755

Ruiqiang Tao

South China Normal University

Guo Tian

South China Normal University

Deyang Chen

South China Normal University

\section{Zhipeng Hou}

South China Normal University

minghui Qin

South China Normal University

\section{Min Zeng}

South China Normal University

\section{Xubing Lu}

South China Normal University https://orcid.org/0000-0002-2552-9571

\section{Guofu Zhou}

South China Normal University

\section{Xingsen Gao}


South China Normal University https://orcid.org/0000-0002-2725-0785

\section{Jun-Ming Liu}

Nanjing University https://orcid.org/0000-0001-8988-8429

\section{Article}

Keywords: ferroelectric photosensor network, in-sensor computing paradigm, ferroelectric photovoltaics

Posted Date: October 25th, 2021

DOI: https://doi.org/10.21203/rs.3.rs-969097/v1

License: (9) This work is licensed under a Creative Commons Attribution 4.0 International License. Read Full License

Version of Record: A version of this preprint was published at Nature Communications on March 31st, 2022. See the published version at https://doi.org/10.1038/s41467-022-29364-8. 


\section{Ferroelectric photosensor network: an advanced hardware solution to real-time machine}

vision

Boyuan Cui ${ }^{1}$, Zhen Fan ${ }^{1 *}$, Wenjie $\mathrm{Li}^{1}$, Yihong $\mathrm{Chen}^{1}$, Shuai Dong ${ }^{1}$, Zhengwei Tan ${ }^{1}$, Shengliang Cheng ${ }^{1}$, Bobo Tian ${ }^{2}$, Ruiqiang Tao ${ }^{1}$, Guo Tian ${ }^{1}$, Deyang Chen ${ }^{1}$, Zhipeng Hou ${ }^{1}$, Minghui Qin ${ }^{1}$, Min Zeng ${ }^{1}$, Xubing Lu ${ }^{1}$, Guofu Zhou ${ }^{3}$, Xingsen Gao ${ }^{1}$, and Jun-Ming Liu ${ }^{4}$

${ }^{1}$ Institute for Advanced Materials and Guangdong Provincial Key Laboratory of Optical Information Materials and Technology, South China Academy of Advanced Optoelectronics, South China Normal University, Guangzhou 510006, China

${ }^{2}$ Key Laboratory of Polar Materials and Devices, Ministry of Education, East China Normal University, Shanghai 200241, China

${ }^{3}$ National Center for International Research on Green Optoelectronics, South China Normal University, Guangzhou 510006, China

4 Laboratory of Solid State Microstructures and Innovation Center of Advanced Microstructures, Nanjing University, Nanjing 210093, China

*Email: fanzhen@m.scnu.edu.cn

[Abstract] Nowadays the development of machine vision is oriented toward real-time applications such as autonomous driving. This demands a hardware solution with low latency, high energy efficiency, and good reliability. Here, we demonstrate a robust and self-powered in-sensor computing paradigm with a ferroelectric photosensor network (FE-PS-NET). The FEPS-NET, constituted by ferroelectric photosensors (FE-PSs) with tunable photoresponsivities, is capable of simultaneously capturing and processing images. In each FE-PS, self-powered photovoltaic responses, modulated by remanent polarization of an epitaxial ferroelectric $\mathrm{Pb}\left(\mathrm{Zr}_{0.2} \mathrm{Ti}_{0.8}\right) \mathrm{O}_{3}$ layer, show not only multiple nonvolatile levels but also a sign reversibility, enabling the representation of a signed weight in a single device and hence reducing the hardware overhead for network construction. With multiple FE-PSs wired together, the FE-PSNET acts on its own as an artificial neural network. It is demonstrated that an in situ multiplyaccumulate operation between an input image and a stored photoresponsivity matrix is available in our FE-PS-NET hardware. The FE-PS-NET hardware is faultlessly competent for real-time image processing functionalities, including binary pattern classification with an accuracy of $100 \%$ and edge detection with an F-Measure of 95.2\%. This study highlights the great potential of ferroelectric photovoltaics as the hardware basis of real-time machine vision. 


\section{Introduction}

Machine vision is a technology that enables a machine to 'see' and 'understand' images and videos, which has been widely applied in industry and daily life. In the conventional design of machine vision systems (Fig. 1a), visual information is captured by a photosensor array, converted into electrical digital signals, and passed to a computing unit for image processing ${ }^{1,2}$. The shuttling of redundant data between separated image sensing and processing units can cause high latency and energy consumption, greatly limiting the performance of machine vision in time-critical applications, such as autonomous driving and object tracking. Emerging bioinspired neuromorphic visual systems (Fig. 1b) provide an opportunity to overcome this limitation $^{3}$. These systems adopt either near- or in-sensor computing architecture (Fig. 1c and $\mathrm{d}$, respectively) to reduce the data shuttling ${ }^{4}$, thus improving the time and energy efficiencies.

So far, a variety of neuromorphic visual systems have been developed for implementing typical image processing functionalities including the contrast enhancement, noise suppression, adaptive imaging, recognition, and auto-encoding ${ }^{5-15}$. Among these systems, reconfigurable photosensor network (PS-NET) with an in-sensor computing architecture (Fig. 1d) is of particular interest because it acts on its own as an artificial neural network (ANN) that can simultaneously sense and process images ${ }^{5,7}$. The key building block for reconfigurable PSNETs is the photosensor with tunable photoresponsivity. Existing designs for such photosensors mainly employed the gating effect in $2 \mathrm{D}$ materials ${ }^{5,7}$ and the ion migration in memristive materials ${ }^{11,12,15}$ to realize the tunable photoresponsivity. However, the required application of gate voltage inevitably consumes additional powers ${ }^{16}$, while the ion migration is kinetically slow and the ion relaxation may cause poor retention ${ }^{11,17}$. New types of reconfigurable photosensors with improved speed, energy efficiency, and reliability are therefore highly desirable.

Ferroelectric photosensor (FE-PS) emerges as an advanced reconfigurable photosensor with all above desired performance. Using the remanent polarization to tune the photovoltaic response $^{18-23}$, the FE-PS is essentially a gate voltage-free and self-powered reconfigurable photosensor ${ }^{24-28}$. Notably, the polarization switching can induce not only the magnitude change but also the sign reversal of photoresponse ${ }^{19,21}$, enabling a single FE-PS to represent both positive and negative weights and hence reducing the hardware overhead for network construction. Moreover, the nonvolativity, high controllability, and ultrafast switching kinetics $(<1 \mathrm{~ns})$ of polarization as demonstrated in various ferroelectric memory and neuromorphic devices $^{29-34}$, along with the intimate coupling between polarization and photoresponse ${ }^{35}$, endow 
the FE-PS with good reliability and high write speed. Also noteworthy are the high photosensitivity and ultrashort photoresponse time $(<1 \mathrm{~ns})$ of FE-PS ${ }^{24,25}$, allowing a high-speed readout. Given the above merits of FE-PS, the FE-PS network (FE-PS-NET), a computing-insensor circuit built with interconnected multiple FE-PSs (Fig. 1d), appears very promising as a fast, low-power, and reliable hardware solution to real-time machine vision. However, while the ferroelectric neuromorphic devices with the memory-computing integrated paradigm have been extensively investigated recently, the FE-PS-NET, representing the first extension to the sensing-memory-computing integrated paradigm, remains experimentally unexplored yet.

Here, we demonstrate a prototype FE-PS-NET with integrated image sensing and processing functions. Each FE-PS in the network consists of a $\mathrm{Pt} / \mathrm{Pb}\left(\mathrm{Zr}_{0.2} \mathrm{Ti}_{0.8}\right) \mathrm{O}_{3}$ $(\mathrm{PZT}) / \mathrm{SrRuO}_{3}(\mathrm{SRO})$ heterostructure epitaxially grown on a $\mathrm{SrTiO}_{3}$ (STO) substrate. The highquality epitaxial PZT film is chosen as the ferroelectric layer for FE-PS because of its large remanent polarization as well as strong and highly controllable photoresponse (albeit in the UV spectrum $)^{21,36}$. SRO is used as the bottom electrode and it also facilitates the epitaxial growth of PZT. The fabricated PZT-based FE-PS exhibits symmetrically switchable, nonvolatile, and multilevel photovoltaic responses as controlled by the remanent polarization. These unique properties enable the FE-PS to be a highly reliable and self-powered reconfigurable photosensor capable of exhibiting both positive and negative photoresponsivities (i.e., weights). Multiple individual FE-PSs are then wired into a FE-PS-NET, whose capability of performing an in situ multiply-accumulate (MAC) operation between an input image and a photoresponsivity matrix is experimentally evidenced. The FE-PS-NET is further used to implement real-time image processing functionalities, including binary pattern classification with an accuracy of $100 \%$ and edge detection with an F-Measure of 95.2\%. Moreover, the ultralow latency and zero-energy consumption for inference are prospected for the FE-PS-NET, underscoring its potential as a hardware platform for real-time machine vision.

\section{Results}

Tunable nonvolatile photoresponsivity in FE-PS. The designed FE-PS has a simple twoterminal structure of Pt/PZT/SRO, as schematically illustrated in Fig. 2a. The PZT/SRO bilayer film was epitaxially grown on the STO (001) substrate by pulsed laser deposition (PLD). The Pt top electrodes were deposited ex situ by sputtering through a shadow mask (diameter: $\sim 200$ $\mu \mathrm{m})$. The low-magnification transmission electron microscopy (TEM) image and X-ray diffraction (XRD) pattern of the fabricated PZT/SRO/STO heterostructure are shown in Supplementary Fig. S1, revealing that both PZT $(\sim 120 \mathrm{~nm})$ and SRO $(\sim 40 \mathrm{~nm})$ layers are well 
formed and exhibit typical perovskite phases. The atomic force microscopy (AFM) image of the PZT/SRO film shows a flat surface with a small root-mean-square roughness of $\sim 470 \mathrm{pm}$ (Fig. 2b). The high-magnification cross-sectional TEM image further reveals the well-aligned lattice of PZT (Fig. 2c). These characterizations demonstrate the high quality of the epitaxial PZT film, which is a prerequisite for obtaining good ferroelectric and photovoltaic properties.

The ferroelectric properties of the Pt/PZT/SRO device were investigated by measuring the bipolar and monopolar polarization-voltage $(P-V)$ hysteresis loops using triangular pulses (pulse width: $0.15 \mathrm{~ms}$ ). The voltage was applied to the Pt electrode with the SRO electrode grounded. Figure $2 \mathrm{~d}$ shows the pulse voltage $\left(V_{\mathrm{p}}\right)$-dependent bipolar $P$ - $V$ loops. The loop starts to open as $V_{\mathrm{p}}$ exceeds $1.8 \mathrm{~V}$ and becomes almost saturated when $V_{\mathrm{p}}$ reaches $2.6 \mathrm{~V}$. The saturated $P-V$ loops reveal a large remanent polarization up to $\sim 80 \mu \mathrm{C} / \mathrm{cm}^{2}$, a typical polarization value of high-quality epitaxial PZT films ${ }^{21,36,37}$. Another key feature of the saturated loops is the negligible voltage offset, namely, the positive and negative coercive voltages are almost symmetric, suggesting that there is only small or even no imprint field. Because the imprint field typically originates from the defects ${ }^{38,39}$, its absence in turn verifies the high quality of our epitaxial PZT film. Besides, the absence of imprint field contributes to the symmetry of switchable photovoltaic responses ${ }^{38,40}$ (to be shown later).

Figure $2 \mathrm{~d}$ also displays that multiple intermediate polarization states are accessible when $V_{\mathrm{p}}$ is in the range of 1.8 to $2.6 \mathrm{~V}$. To confirm it, monopolar triangular pulses with different $V_{\mathrm{p}}$ were applied and the measured $P-V$ loops are shown in Fig. 2e and f. Every time before applying the measurement pulse, a $-3 \mathrm{~V}$ or $+3 \mathrm{~V}$ preset pulse was applied to set the complete polarization up $\left(P_{\text {up }}\right)$ or down $\left(P_{\text {down }}\right)$ state, respectively. As seen from Fig. 2e, when starting from the same complete $P_{\text {up }}$ state $\left(\sim-80 \mu \mathrm{C} / \mathrm{cm}^{2}\right)$, applying positive pulses with $V_{\mathrm{p}} \leq+1.8 \mathrm{~V}$ makes almost no change in the polarization state. Applying positive pulses with $V_{\mathrm{p}}=+2,+2.2$, and $+2.4 \mathrm{~V}$ results in three well-separated intermediate states: incomplete $P_{\text {up }}$, near-zero-polarization, incomplete $P$ down states, whose corresponding remanent polarization values are $\sim-40, \sim 0$, and $\sim+40 \mu \mathrm{C} / \mathrm{cm}^{2}$, respectively. Further increasing $V_{\mathrm{p}}$ to $+2.6 \mathrm{~V}$ and above switches the device to the complete $P_{\text {down }}$ state $\left(\sim+80 \mu \mathrm{C} / \mathrm{cm}^{2}\right)$. Likewise, by applying negative pulses with increasing $V_{\mathrm{p}}$, the complete $P_{\text {down }}$ state is switched to the incomplete $P_{\text {down }}$ state, near-zero-polarization state, incomplete $P_{\text {up }}$ state and eventually complete $P_{\text {up }}$ state (Fig. 2f). In addition, the loops in Fig. $2 \mathrm{e}$ and $\mathrm{f}$ exhibit relatively flat tops and bottoms, respectively, indicating that the polarizations can be retained when the external voltages return to zero. This implies that the polarization states, including the intermediate states, are nonvolatile. 
The formation mechanism of intermediate polarization states was investigated by using piezoresponse force microscopy (PFM). Supplementary Fig. S2 shows that the downward (upward) domains can be gradually switched upward (downward) as the applied negative (positive) tip voltage increases. In particular, upward/downward mixed domain configurations are observed when medium tip voltages are applied, giving rise to intermediate polarization states. All the domain states are found to be stable (up to 18 days of retention), confirming the nonvolatility of the polarization states. Such good domain stability may benefit from the domain growth-dominated switching behavior, as discussed in Supplementary Fig. S2.

Given the excellent ferroelectric properties of the present epitaxial PZT film (including large remanent polarization, negligible voltage offset, and accessibility to multiple nonvolatile polarization states), the polarization-modulated photovoltaic behavior in the PZT-based FE-PS is worthy of investigation. To characterize it, monopolar triangular pulses were applied first to set the polarization states, as illustrated in Fig. 2e and f. In each polarization state, currentvoltage $(I-V)$ characteristics under illumination were recorded by using the $365 \mathrm{~nm}$ UV light for illumination, because this light wavelength corresponds well to the bandgap of PZT ( 3.6 $\mathrm{eV})^{36}$. Unless otherwise specified, the applied light intensity was $\sim 150 \mathrm{~mW} / \mathrm{cm}^{2}$ (corresponding optical power was $\sim 47.1 \mu \mathrm{W}$ for an electrode area of $\sim 0.0314 \mathrm{~mm}^{2}$ ). Figure $2 \mathrm{~g}$ shows the illuminated $I-V$ curves of the FE-PS in the different polarization states as set by the different positive pulses. In the initial $-3 \mathrm{~V}$-written state, the FE-PS exhibits noticeable photovoltaic responses including a short-circuit current $\left(I_{\mathrm{sc}}\right)$ of $\sim 10.6 \mathrm{nA}$ and an open-circuit voltage $\left(V_{\mathrm{oc}}\right)$ of $\sim-0.5 \mathrm{~V}$. Such $I_{\mathrm{sc}}$ is 3 orders of magnitude larger than the dark current $(\sim-0.02 \mathrm{nA}$ at $-0.5 \mathrm{~V})$. The illuminated $I-V$ curve as well as $I_{\mathrm{sc}}$ and $V_{\mathrm{oc}}$ remains almost unchanged after applying positive pulses with $V_{\mathrm{p}} \leq+1.8 \mathrm{~V}$. After applying the $+2 \mathrm{~V}$ pulse, the illuminated $I-V$ curve shifts toward the origin, and $I_{\mathrm{sc}}$ and $V_{\mathrm{oc}}$ decrease to $\sim 5.4 \mathrm{nA}$ and $\sim-0.25 \mathrm{~V}$, respectively. As $V_{\mathrm{p}}$ increases to $+2.2 \mathrm{~V}$, the illuminated $I-V$ curve moves very close to the origin, showing nearzero values of $I_{\mathrm{sc}}(\sim 0.7 \mathrm{nA})$ and $V_{\mathrm{oc}}(\sim-0.04 \mathrm{~V})$. Increasing $V_{\mathrm{p}}$ to $+2.4 \mathrm{~V}$ pushes the illuminated $I-V$ curve away from the origin along the positive voltage and negative current axes. As a result, both $I_{\mathrm{sc}}$ and $V_{\mathrm{oc}}$ change their signs (i.e., directions), and their values are $\sim-4.4 \mathrm{nA}$ and $\sim 0.28 \mathrm{~V}$, respectively. The illuminated $I-V$ curve is further pushed away from the origin after applying the $+2.6 \mathrm{~V}$ pulse, and $I_{\text {sc }}$ and $V_{\text {oc }}$ become $\sim-9.9 \mathrm{nA}$ and $\sim 0.5 \mathrm{~V}$, respectively. Further increasing $V_{\mathrm{p}}$ to $+2.8 \mathrm{~V}$ and above makes no more change in the illuminated $I-V$ curve. There are thus five photoresponsive states observed during the switching process (note: a much larger number of states are indeed accessible, to be shown in Fig. 3). Apparently, these photoresponsive states 
show almost one-to-one correlation with the polarization states (Fig. 2e), demonstrating that the photoresponse is well controlled by the polarization.

In addition, the reverse switching of the photoresonsive state is observed by applying negative pulses (see Fig. 2h), which is consistent with the down-to-up polarization switching (Fig. 2f). Plots of $I_{\mathrm{sc}}$ and $V_{\mathrm{oc}}$ against the pulse voltage $V_{\mathrm{p}}$ form well-shaped hysteresis loops akin to the $P-V$ hysteresis loops (Fig. 2i), further confirming the reversible polarization control of photovoltaic response. Tunable photoresponse obtained in the photovoltaic mode qualifies the FE-PS as a self-powered reconfigurable photosensor. In addition, unlike previous photovoltaictype reconfigurable photosensors ${ }^{5}$, the FE-PS does not need to use the gate voltage to switch the photoresponse. It uses the remanent polarization as the control knob instead, which can further lower the power consumption.

Figure $2 \mathrm{~g}$ and h also shows that in a pair of $P_{\text {up }}$ and $P_{\text {down }}$ states with opposite remanent polarizations, the two $I_{\mathrm{sc}}$ (or $V_{\mathrm{oc}}$ ) values have opposite signs but similar magnitudes. For example, the $I_{\mathrm{sc}}$ value in the complete $P_{\text {up }}$ state is $\sim 10 \mathrm{nA}$, while that in the complete $P_{\text {down }}$ state is just the opposite, i.e., $\sim 10 \mathrm{nA}$. Such symmetry of switchable photoresponses is a result of the dominated polarization control, which benefits from the high-quality epitaxial PZT film possessing large switchable polarization and negligible imprint field (see evidence from the symmetric $P-V$ loops in Fig. 2d) $)^{38,40}$. From the application point of view, symmetrically switchable photoresponses enable a single FE-PS to represent both positive and negative weights, which is particularly useful for reducing the number of FE-PSs needed for network construction.

Because the photovoltaic behavior is controlled by the polarization without defectmediated mechanisms (e.g., ion migration) involved, good reliability is expected for FE-PS. We first investigated the stability of the polarization-controlled photoresponses. As shown in Supplementary Fig. S3, the photocurrents in all the representative polarization states are stable with a rather long retention time of $\geq 24$ hours, and they are reproducible during frequent ON/OFF illumination cycling. This demonstrates the nonvolatility of the photoresponsive states, which benefits from the nonvolativity of the polarization states (Fig. 2d-f and Supplementary Fig. S2). Then, the endurance test was performed by switching the FE-PS with cyclic $3 \mathrm{~V} / 10$ $\mu$ s pulses. Supplementary Fig. S4 shows that both the photocurrents and associated remanent polarizations change only slightly after switching for $10^{6}$ cycles, highlighting the good endurance of the FE-PS. Device-to-device variation was characterized by measuring the $P-V$ loops and photocurrents of 11 different FE-PSs (Supplementary Fig. S5). These devices all 
exhibit switchable photocurrents, and the photocurrents in the same polarization state show a small variation of $\sim 3.2 \%$. In addition, to enable the FE-PS to perform the multiplication (i.e., photosensing) reliably, a linear dependence of photocurrent on light intensity is required. As shown in Supplementary Fig. S6, the photocurrents in different polarization states scale almost linearly with the light intensity (linearity: $\geq 0.94$ ), thus satisfying the requirement of multiplication.

The polarization control of photovoltaic behavior with high reliability promises our FEPS as a superior synaptic device (using the photoresponsivity as the weight). To demonstrate it, typical synaptic behaviors, i.e., long-term potentiation and depression (LTP and LTD, respectively), were measured for the FE-PS. In the measurement, a positive pulse train (amplitude: from $1.65 \mathrm{~V}$ to $1.89 \mathrm{~V}$ in increments of $0.01 \mathrm{~V}$; width: $10 \mu \mathrm{s}$ ) and a negative pulse train (amplitude: from $-1.75 \mathrm{~V}$ to $-1.99 \mathrm{~V}$ in decrements of $0.01 \mathrm{~V}$; width: $10 \mu \mathrm{s}$ ) were applied alternately to the FE-PS (see inset in Fig. 3a). The pulse voltages were slightly below the coercive voltages and increased in magnitude so that the polarization could be switched gradually to produce many intermediate states (note: the coercive voltages of the device used for the LTP/LTD measurement are $\sim \pm 1.9 \mathrm{~V}$ ). After each positive or negative pulse, $I_{\mathrm{sc}}$ was measured and used to calculate the photoresponsivity defined as

$R=I_{\mathrm{sc}} / P$,

where $R$ is the photoresponsivity and $P$ is the input optical power (product of light intensity and electrode area). As shown in Fig. 3a, $R$ decreases gradually from $\sim 0.22 \mathrm{~mA} / \mathrm{W}$ to $\sim-0.22 \mathrm{~mA} / \mathrm{W}$ with increasing the number of positive pulses, indicating the LTD behavior. By contrast, $R$ increases from $\sim-0.22 \mathrm{~mA} / \mathrm{W}$ back to $\sim 0.22 \mathrm{~mA} / \mathrm{W}$ under the stimulation of negative pulses, a manifestation of the LTP behavior. Similar LTD and LTP characteristics can be reproduced for many cycles (Fig. 3b), showing a small cycle-to-cycle variation of $\sim 3 \%$. Notably, each LTD or LTP process contains 25 different $R$ levels, confirming the accessibility to multiple photoresponsive states. One may further achieve a larger number of $R$ levels by manipulating the applied pulses.

The integrated synaptic and photosensing functions of the FE-PS, as demonstrated above, allows the construction of FE-PS-NET with in-sensor computing capability. Prior to constructing it, the physical mechanism underlying the polarization control of photovoltaic behavior in the FE-PS needs to be understood. We previously demonstrated that the polarization-modulated Schottky barrier was responsible for the switchable photovoltaic behavior in the Pt/PZT/SRO FF-PS, through comprehensive investigations on the ferroelectric, 
dielectric, conduction, and photovoltaic behaviors of the device ${ }^{36}$. In brief, epitaxial PZT film which is an $n$-type semiconductor ${ }^{41}$ can form Schottky barriers with Pt and SRO. Assuming that there is no polarization in PZT, the top Pt/PZT and bottom PZT/SRO barriers would have similar heights due the similar work functions of Pt and $\operatorname{SRO}(\sim 5.3$ and $\sim 5.2 \mathrm{eV}$, respectively). However, the polarization of PZT can significantly modify the barrier heights at the top and bottom interfaces as well as the associated built-in fields ( $E_{\mathrm{bi}-\mathrm{t}}$ and $E_{\mathrm{bi}-\mathrm{b}}$, respectively). In the complete $P$ up state, the negative polarization charge at the PZT/SRO interface enhances the bottom barrier height and $E_{\mathrm{bi}}$, while the positive polarization charge at the Pt/PZT interface

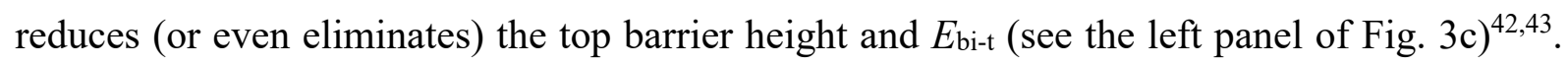
The downward $E_{\text {bi-b }}$ therefore dominates and generates an overall positive photocurrent. By contrast, the dominance of $E_{\mathrm{bi}-\mathrm{t}}$ occurs in the complete $P_{\text {down }}$ state, producing an overall negative photocurrent (see the right panel of Fig. 3c). In the intermediate polarization states, the relative proportion of upward and downward domains may determine the magnitude and direction of overall photocurrent, and hence multilevel photocurrents are accessible. The Schottky barrier modulation can therefore well explain the polarization-controlled switchable photoresponse in the Pt/PZT/SRO FE-PS.

In-sensor MAC operations in FE-PS-NET. Having demonstrated the switchable photoresponsivity of FE-PS and understood its physical mechanism, it is of interest to investigate the hardware implementation of MAC (a fundamental operation for the simultaneous image sensing and processing) using FE-PS-NET. As schematically shown in Fig. $4 \mathrm{a}$ and $\mathrm{b}$, the FE-PS-NET consists of $N$ pixels with each pixel divided into $M$ subpixels. $N$ depends on the image size, i.e., $N=H \times W$, where $H$ and $W$ are the height and width of the image, respectively, and the $N$ pixels are arranged in an $H \times W$ array to suit the image. The $M$ subpixels are also arranged in a 2D array for saving the area overhead. Each subpixel corresponds to a FE-PS, which has a subpixel index $(m=1,2, \ldots, M)$ as well as a pixel index $(n=1,2, \ldots, N)$. The FE-PSs with the same subpixel index $m$ are connected in parallel. With such an architecture, the FE-PS-NET can perform an efficient in-sensor MAC operation: under short-circuit and illumination conditions, the multiplication of optical power and photoresponsivity occurs at each individual FE-PS through the photosensing process; meanwhile, the photocurrents generated by the $N$ FE-PSs with the same subpixel index $m$ are summed together according to the Kirchhoff's law. The output current $I_{m}$ is expressed as

$$
I_{m}=\sum_{n=1}^{N} R_{m n} P_{n}
$$


where $R_{m n}$ is the photoresponsivity of the FE-PS at the $n$-th pixel and $m$-th subpixel [denoted as the $(m, n)$ FE-PS hereafter], and $P_{n}$ is the input optical power at the $n$-th pixel [the vector $\mathbf{P}_{\text {in }}=$ $\left(P_{1}, P_{2}, \ldots, P_{N}\right)^{\mathrm{T}}$ represents the input image].

To experimentally demonstrate the MAC, i.e., Eq (2), a simple $1 \times 2(M=1$ and $N=2)$ FE-PS-NET was used first (see Fig. 4c). Both of the two FE-PSs were set in the complete $P_{\text {up }}$ states beforehand. The resulting photoresponsivities $R_{11}$ and $R_{12}$ were thus almost the same, i.e., $\sim 0.22 \mathrm{~mA} / \mathrm{W}$. As shown in Fig. 4d, during the first 30 seconds, no illumination is applied and zero output current, i.e., $I_{1}$, is observed. During the period of 30 to 60 seconds, the $(1,1)$ FE-PS is illuminated with an optical power of $P_{1}=\sim 4.5 \mu \mathrm{W}$, resulting in a photocurrent of $\sim 1 \mathrm{nA}$. During the next 30 seconds, both two FE-PSs are illuminated with $P_{1}=P_{2}=\sim 4.5 \mu \mathrm{W}$. The output current jumps to $\sim 2 \mathrm{nA}$, which is just the summation of the photocurrents generated by the two FE-PSs. Then, the illumination on the $(1,1)$ FE-PS is turned off while that on the $(1,2)$ FE-PS remains. The output current drops to $\sim 1 \mathrm{nA}$, which is the photocurrent generated by the individual $(1,2)$ FE-PS. After this, the illuminations on both two FE-PSs are turned off, and consequently the output current returns to zero. During the periods of 150 to 240 and 270 to 360 seconds, another two rounds of illuminations are applied. These two rounds of illuminations have the same sequence of applying $P_{1}$ and $P_{2}$ as the first round (30 to 120 seconds). However, the magnitudes of $P_{1}$ and $P_{2}$ are adjusted: $P_{1}=\sim 13.5 \mu \mathrm{W}$ and $P_{2}=\sim 4.5$ $\mu \mathrm{W}$ in the second round while $P_{1}=\sim 13.5 \mu \mathrm{W}$ and $P_{2}=\sim 13.5 \mu \mathrm{W}$ in the third round. Inspecting the periods where only one individual FE-PS is illuminated, one can find that the photocurrent of the individual FE-PS scales with the optical power, confirming the validity of multiplication. Besides, it is observed that the output currents during the periods where both two FE-PSs are illuminated always equal the summed photocurrents of the two FE-PSs.

After these measurements, the $(1,1)$ FE-PS was set in the complete $P_{\text {down }}$ state while no change of polarization state was made for the $(1,2)$ FE-PS. The resulting photoresponsivities $R_{11}$ and $R_{12}$ were thus $\sim-0.22 \mathrm{~mA} / \mathrm{W}$ and $\sim 0.22 \mathrm{~mA} / \mathrm{W}$, respectively. Then, three rounds of illuminations same as those used in Fig. $4 \mathrm{~d}$ were applied again to the $1 \times 2$ FE-PS-NET, and the output currents are shown in Fig. 4e. The photocurrent generated by the $(1,1)$ FE-PS is observed to be negative, well attributed to the negative $R_{11}$. Moreover, both multiplication and summation operations are observed to be valid. The combined Fig. $4 \mathrm{~d}$ and e therefore demonstrate that the $1 \times 2$ FE-PS-NET can perform the MAC operations following Eq. (2).

Whether Eq. (2) still applies in a FE-PS-NET with larger size remains a question because the sneak path issue may arise. The sneak path issue refers to the unintentional current flow 
through neighboring unselected devices in a crossbar structure. It is a common issue encountered by conventional memristor crossbar-based $\mathrm{ANNs}^{44}$, which can cause significant errors in output currents. The sneak path issue in our FE-PS-NET was investigated with a $2 \times$ $2(M=2$ and $N=2)$ FE-PS-NET, as schematically shown in Fig. 4f. All the four FE-PSs were set in the complete $P_{\text {up }}$ state, resulting in almost the same photoresponsivity of $\sim 0.22 \mathrm{~mA} / \mathrm{W}$. The output current $I_{1}$ was monitored while applying a sequence of illuminations to the four FEPSs. The illuminations for the four FE-PSs were applied or terminated independently, and the optical power during illumination was $\sim 25 \mu \mathrm{W}$. As seen from in Fig. $4 \mathrm{~g}$, applying or removing illuminations to the $(2,1)$ and $(2,2)$ FE-PSs have almost no influences on the multiplication and summation operations performed by the sub-circuit composed of the $(1,1)$ and $(1,2) \mathrm{FE}$ PSs. Therefore, our FE-PS-NET has good immunity to the sneak path issue. The reason for this may be because the FE-PS-NET works under the short-circuit condition and the illumination rather than the bias is used to select the device. The photocurrent generated by a selected device would therefore not flow through a neighboring unselected device. Even in the case where the short-circuit condition is not strictly met and a small bias arising from the photovoltaic effect of a selected device does drop across a neighboring unselected device, the leakage current produced by the neighboring unselected device would be negligible due to its high resistance (see Fig. $2 \mathrm{~g}$ and h). This is fundamentally different from the scenario in a memristor crossbar, where the neighboring unselected device in the $\mathrm{ON}$ state can contribute a large leakage current. Therefore, the good immunity of FE-PS-NET to the sneak path issue is confirmed, allowing it to perform the MAC operations properly even with a large-scale network.

Implementations of pattern classification and edge detection. With the capability to perform in-sensor MAC operations, the FE-PS-NET can readily be used to implement real-time image processing functionalities. Pattern classification was demonstrated first. Two sets of patterns, representing the letters ' $\mathrm{X}$ ' and ' $\mathrm{T}$ ' and their variants after adding noises (Fig. 5a), were used as both training and test sets ${ }^{45,46}$. Each pattern contained $3 \times 3=9$ pixels, and the pixel values of black and white pixels were defined as 1 and 0 , respectively. The classes of ' $\mathrm{X}$ ' and ' $\mathrm{T}$ ' corresponded to the binary outputs of 1 and 0 , respectively. Such pattern classification task was solvable by a single-layer perceptron containing 9 input neurons and 1 output neurons. The single-layer perceptron was hardware implemented with a $1 \times 9(M=1$ and $N=9)$ FE-PS-NET (see Fig. 5b). When presenting an input pattern to the FE-PS-NET, the pixel value of 1 or 0 at a specific pixel represented applying or removing illumination (optical power: $\sim 4.5 \mu \mathrm{W}$ ) to the corresponding FE-PS, respectively. Through the MAC process, the FE-PS-NET produced an output current $I_{1}$. $I_{1}$ was further fed to a sigmoid activation function to generate a neuronal 
output (see Methods). The sigmoid activation function was implemented in software here, but it could be implemented with conventional CMOS circuits ${ }^{47}$. Training was also performed in software, a method called the ex situ training. Then, the calculated weight matrix was transferred to the FE-PS-NET. When programming each FE-PS, a write-and-verify method was used to ensure a small discrepancy between the actual and target photoresponsivities. The FEPS-NET after programming could conduct the inference once an input pattern was presented to it.

Figure $5 \mathrm{c}$ compares the calculated and actual weight matrices. The differences between the actual weights and the corresponding calculated ones are quite small, indicating the successful programming of the FE-PS-NET. Figure 5d shows the output current $I_{1}$ during the presentations of different input patters. It is observed that the output current is always positive when a pattern belonging to the ' $\mathrm{X}$ ' class is presented, while it is always negative when a pattern belonging to the ' $\mathrm{T}$ ' class is presented. Moreover, the measured output currents agree well with the theoretically calculated ones. Figure 5e further presents the neuronal outputs derived from the output currents. The neuronal outputs of the ' $\mathrm{X}$ '-class patterns are all close to 1 while those of the ' $\mathrm{T}$ '-class patterns are all close to 0 , demonstrating that all the patterns are correctly classified. The accuracy for this simple binary classification task is therefore $100 \%$. After the pattern classification, the weights exhibit only slight changes (Fig. 5c), demonstrating good reliability of the FE-PS-NET as a pattern classifier.

Another important image processing functionality, i.e., the edge detection, was also demonstrated. As shown in Fig. 6a, A $11 \times 11$ image showing an arrow sign was used as the input image. The pixel values in this image were binarized, similar as those used for the pattern classification. For the convolution operation in the edge detection, $3 \times 3$ kernels were used to slide over the input image with a stride of 1 . Consequently, the initial input image was decomposed into $813 \times 3$ sub-images. These sub-images, with pixel values translated to illumination signals, were presented sequentially to the kernels based on FE-PS-NET. The kernels used here were two Sobel kernels, as displayed in Fig. 6b. These two kernels were implemented with a $2 \times 9(M=2$ and $N=9)$ FE-PS-NET. The kernel weights were mapped to the photoresponsivities of the corresponding FE-PSs (see Fig. 6b). During the convolution, the dot product between a sub-image and a kernel was obtained through the MAC process in the FE-PS-NET (see Fig. 6a). After the convolution, two sets of output current data $I_{1}$ and $I_{2}$, corresponding to Kernel 1 and 2, respectively, were collected. They were further merged, normalized, and binarized to form the output image (see Methods). 
Figure $6 \mathrm{c}$ shows the output currents $I_{1}$ and $I_{2}$ after the convolution with the two kernels. All the actual current values agree well with the theoretically calculated ones. Figure $6 \mathrm{~d}$ (right panel) presents the final output image, clearly revealing the edge between the arrow and the background (note: the edge contains both the outermost pixels of foreground and innermost pixels of background). Based on Fig. 6d, a performance metric, i.e., F-Measure, is calculated to be $95.2 \%$, quite close to 1 . This demonstrates the good performance of edge detection implemented with the FE-PS-NET. In addition, almost no changes are observed in the kernel weights after the edge detection (Fig. 6b), verifying the reliability of the FE-PS-NET as an edge detector.

The high accuracy and reliability of FE-PS-NET for image processing can be well attributed to the reliable polarization control of photoresponsivity, as demonstrated previously. In addition, the low latency is another merit of FE-PS-NET because it can simultaneously sense and process images in the analog domain. The operation speed is thus limited mainly by the photocurrent generation time and the RC time constant of the circuit. Due to time resolution limit of our measurement system, we can only confirm that the photocurrent generation time is below 100 ms (Supplementary Fig. S7). Indeed, the photocurrent generation in a FE-PS can occur within $1 \mathrm{~ns}^{24,25}$; hence, the RC time constant of the circuit may become the major speedlimiting factor. A rough estimation shows that the total latency of sensing and processing a 10million-pixel image is $\sim 2.6 \mu$ s for the FE-PS-NET, which is 4 orders of magnitude shorter than that of a conventional Von Neumann system (Supplementary Note 1). In terms of the energy consumption, because the FE-PS operates in the gate voltage-free photovoltaic (i.e., selfpowered) mode, zero energy is in principle consumed when performing the inference. The energy is consumed only when programing the FE-PS. As shown in Supplementary Fig. S8, applying $\pm 2 \mathrm{~V} / 10 \mu$ s programing pulses to our $\sim 0.0314 \mathrm{~mm}^{2}$ PZT-based FE-PS results in an energy consumption of $\sim 3.1 \mathrm{~nJ}$. As the FE-PS could be scaled down to $\sim 1 \mu^{2}{ }^{48}$, the energy consumption may thus be reduced to $\sim 0.1 \mathrm{pJ}$ per bit per operation, which is a sufficiently low value compared with those of recent emerging neuromorphic devices ${ }^{14,32}$. The good scalability also allows the construction of a large-scale FE-PS-NET in a small area. The area efficiency could further benefit from the following two factors. First, the FE-PS-NET store the weights locally, and thus no external memory is needed to remember the weights. In addition, a single FE-PS can represent both positive and negative weights, making it unnecessary to use a pair of FE-PSs to represent a signed weight. The above features including high speed, scalability, and reliability, as well as low energy consumption, make the FE-PS-NET a good candidate for the hardware implementation of real-time machine vision. 


\section{Discussion}

In summary, we achieved a proof-of-concept demonstration of FE-PS-NET that can simultaneously sense and process images. The FE-PS-NET was constructed by wiring multiple FE-PSs with each FE-PS consisting of a two-terminal Pt/PZT (epitaxial film)/SRO heterostructure. The FE-PS exhibited multilevel nonvolatile photoresponses as well controlled by the remanent polarization. Also benefitting from the polarization control, small cycle-tocycle and device-to-device variations ( $\sim 3 \%$ and $\sim 3.2 \%$, respectively) as well as high endurance $\left(1 \times 10^{6}\right.$ cycles $)$ were demonstrated for the FE-PS. Moreover, the switching of the polarization direction induced the reversal of the photocurrent direction, thus enabling a single FE-PS to represent both positive and negative weights. Using the FE-PS as a building block, the FE-PSNET exhibited the capability to perform in-sensor MAC operations. The FE-PS-NET was further demonstrated with real-time image processing functionalities, including binary pattern classification with an accuracy of $100 \%$ and edge detection with an F-Measure of $95.2 \%$. Moreover, because of the polarization-controlled photovoltaic operation mode, ultrafast photocurrent generation process, and in-sensor computing architecture, the FE-PS-NET could achieve high reliability, ultralow latency, and zero energy consumption for inference. This study demonstrates the first type of ferroelectric neuromorphic device with the sensingmemory-computing integrated paradigm, opening up a new way for the development of reliable, high-speed, and low-power hardware for real-time machine vision.

\section{Methods}

Device fabrication. $\sim 40 \mathrm{~nm}$ SRO and $\sim 120 \mathrm{~nm}$ PZT epitaxial thin films were successively grown on (001)-oriented STO single crystalline substrates by PLD using a $\mathrm{KrF}$ excimer laser $(\lambda=248 \mathrm{~nm})$. An energy fluence of $0.9 \mathrm{~J} / \mathrm{cm}^{2}$ and a repetition rate of $5 \mathrm{~Hz}$ were used for the depositions of both SRO and PZT films. The SRO films were first deposited at a substrate temperature of $680{ }^{\circ} \mathrm{C}$ under an oxygen pressure of $15 \mathrm{~Pa}$. The PZT films were subsequently deposited under the same oxygen pressure, but the substrate temperature was lowered to $600{ }^{\circ} \mathrm{C}$. After growth, the PZT/SRO films were cooled to room temperature at a rate of $10{ }^{\circ} \mathrm{C} / \mathrm{min}$ under $1 \mathrm{~atm}$ oxygen pressure. The Pt top electrodes with $\sim 10 \mathrm{~nm}$ in thickness were ex situ deposited on the films through a shadow mask (diameter: $\sim 200 \mu \mathrm{m}$ ) by PLD at room temperature and under vacuum. The individual Pt/PZT/SRO FE-PSs were thus formed. To construct a FE-PSNET, the individual FE-PSs were connected by wiring. For the wiring, the two ends of a Pt wire (diameter: $50 \mu \mathrm{m}$ ) were pasted onto the two Pt top electrodes intended to be connected by using the silver paste. 
Characterizations. The crystalline structure and phase purity of the films were investigated by XRD (X' Pert PRO, PANalytical). The epitaxial quality and microstructure were further examined using TEM (Tecnai G2-F20). The surface morphology and domain structure were characterized by AFM and PFM, respectively, which were performed on an integrated scanning probe microscope (Asylum Research MFP-3D) with Pt-coated silicon tips (Nanoworld EFM Arrow). The PFM amplitude and phase images were acquired by using an AC driving voltage of $0.8 \mathrm{~V}$ in the DART (dual a.c. resonance tracking) mode.

Electrical measurements. The bipolar and monopolar $P-V$ hysteresis loops were measured with triangular pulses (pulse width: $150 \mu \mathrm{s}$ ) on a ferroelectric workstation (Radiant Precision Multiferroic). The $I-V$ characteristics were measured with a SourceMeter (Keithley 6430). Both the ferroelectric workstation and SourceMeter were used to apply electrical pulses with various amplitudes and widths. In the photovoltaic measurement, a $365 \mathrm{~nm}$ UV light-emitting diode (LED) with tunable light intensities was used as the light source while the SourceMeter recorded the photocurrent data. When applying illumination to an individual FE-PS, the whole electrode area was considered for the calculation of optical power. However, for the FE-PS in FE-PS-NET, only the area without the coverage of the silver paste was considered as being subjected to the illumination and used for the calculation of optical power.

Simulations. For the pattern classification task, the sigmoid activation function which was implemented in software is expressed as:

$$
\begin{aligned}
& f(x)=\frac{1}{1+e^{-x}}, \\
& x=\alpha I_{1},
\end{aligned}
$$

where $x$ is the neuronal input scaled from the measured current $I_{1}$, and $\alpha$ is a scaling factor $(\alpha=$ $3 \mathrm{nA}^{-1}$ in this work).

For the edge detection task, two Sobel kernels were used for the convolution, which are expressed as:

$$
\text { Kernel } 1=\left[\begin{array}{ccc}
1 & \sqrt{2} & 1 \\
0 & 0 & 0 \\
-1 & -\sqrt{2} & -1
\end{array}\right] \text {, }
$$


Kernel $2=\left[\begin{array}{ccc}-1 & 0 & 1 \\ -\sqrt{2} & 0 & \sqrt{2} \\ -1 & 0 & 1\end{array}\right]$.

After the convolution, two maps of output current data ( $I_{1}$ and $I_{2}$, corresponding to Kernel 1 and 2 , respectively) were obtained. The two maps were merged into one map following the equation below:

$I_{\mathrm{E}}=\left|I_{1}\right|+\left|I_{2}\right|$

Then, the $I_{\mathrm{E}}$ values were normalized to the range $[0,1]$. The normalized $I_{\mathrm{E}}$ values were further binarized as follows:

Normalized $I_{E}=\left\{\begin{array}{l}0, x<d \\ 1, x \geq d\end{array}\right.$,

where $d$ is a threshold value, and $d=0.6$ was used in this work.

After the normalization and binarization, the final output image showing the detected edge was obtained. The F-Measure was used to evaluate the quality of the output image, as given by

F-Measure $=\frac{\left(1+\beta^{2}\right) P R}{\beta^{2} P+R}$,

where $\beta$ is a constant ( $\beta=1$ was used here), $P$ is the precision, and $R$ is the recall.

\section{Data availability}

The data that support the findings of this study are available from the corresponding author upon reasonable request.

\section{References}

1. Moini, A. Vision Chips. Springer Science \& Business Media (2012).

2. Lee, G. J., Choi, C., Kim, D. H., \& Song, Y. M. Bioinspired artificial eyes: Optic components, digital cameras, and visual prostheses. Adv. Funct. Mater. 28, 1705202 (2018).

3. Liao, F., Zhou, F., \& Chai, Y. Neuromorphic vision sensors: Principle, progress and perspectives. J. Semicond. 42, 013105 (2021).

4. Zhou, F., \& Chai, Y. Near-sensor and in-sensor computing. Nat. Electron. 3, 664-671 (2020). 
5. Mennel, L., Symonowicz, J., Wachter, S., Polyushkin, D. K., Molina-Mendoza, A. J., \& Mueller, T. Ultrafast machine vision with 2D material neural network image sensors. Nature 579, 62-66 (2020).

6. Zhou, F. et al. Optoelectronic resistive random access memory for neuromorphic vision sensors. Nat. Nanotechnol. 14, 776-782 (2019).

7. Wang, C. et al. Gate-tunable van der Waals heterostructure for reconfigurable neural network vision sensor. Sci. $A d v$. 6, eaba6173 (2020).

8. Wang, S. et al. Networking retinomorphic sensor with memristive crossbar for braininspired visual perception. Natl. Sci. Rev. 8, nwaa172 (2021).

9. Seo, S. et al. Artificial optic-neural synapse for colored and color-mixed pattern recognition. Nat. Commun. 9, 1-8 (2018).

10. Xie, D. et al. Coplanar multigate $\mathrm{MoS}_{2}$ electric-double-layer transistors for neuromorphic visual recognition. ACS Appl. Mater. Interfaces 10, 25943-25948 (2018).

11. Chen, Q. et al. Switchable perovskite photovoltaic sensors for bioinspired adaptive machine vision. Advanced Intelligent Systems 2, 2000122 (2020).

12. Wang, Y. et al. MXene-ZnO Memristor for Multimodal In-Sensor Computing. Adv. Funct. Mater. 31, 2100144 (2021).

13. Zhu, Q. et al. A flexible ultrasensitive optoelectronic sensor array for neuromorphic vision systems. Nat. Commun. 12, 1-7 (2021).

14. Kumar, M., Lim, J., \& Seo, H. Highly Transparent Reconfigurable Non-Volatile Multilevel Optoelectronic Memory for Integrated Self-Powered Brain-Inspired Perception. Nano Energy 89, 106471 (2021).

15. Wang, T. et al. Reconfigurable optoelectronic memristor for in-sensor computing applications. Nano Energy 89, 106291 (2021).

16. Chai, Y. In-sensor computing for machine vision. Nature 579, 32-33 (2020).

17. Ham, S., Choi, S., Cho, H., Na, S. I., \& Wang, G. Photonic Organolead Halide Perovskite Artificial Synapse Capable of Accelerated Learning at Low Power Inspired by DopamineFacilitated Synaptic Activity. Adv. Funct. Mater. 29, 1806646 (2018).

18. Choi, T., Lee, S., Choi, Y. J., Kiryukhin, V., \& Cheong, S. W. Switchable ferroelectric diode and photovoltaic effect in $\mathrm{BiFeO}_{3}$. Science 324, 63-66 (2009).

19. Ji, W., Yao, K., \& Liang, Y. C. Bulk photovoltaic effect at visible wavelength in epitaxial ferroelectric BiFeO3 thin films. Adv. Mater. 22, 1763-1766 (2010).

20. Guo, R. et al. Non-volatile memory based on the ferroelectric photovoltaic effect. Nat. Commun. 4, 1-5 (2013). 
21. Pintilie, L., Vrejoiu, I., Le Rhun, G., \& Alexe, M. Short-circuit photocurrent in epitaxial lead zirconate-titanate thin films. J. Appl. Phys. 101, 064109 (2007).

22. Zhou, Y. et al. Switchable ferroelectric diode and photovoltaic effects in polycrystalline $\mathrm{BiFeO}_{3}$ thin films grown on transparent substrates. Thin Solid Films 698, 137851 (2020).

23. Li, Y., Cui, X., Sun, N., Du, J., Li, X., Jia, G., \& Hao, X. Region-Dependent and Stable Ferroelectric Photovoltaic Effect Driven by Novel In-Plane Self-Polarization in NarrowBandgap Bi2FeMo0. 7Ni0. $3 \mathrm{O}_{6}$ Thin Film. Adv. Opt. Mater. 7, 1801105 (2019).

24. Li, J. K., Ge, C., Jin, K. J., Du, J. Y., Yang, J. T., Lu, H. B., \& Yang, G. Z. Self-driven visible-blind photodetector based on ferroelectric perovskite oxides. Appl. Phys. Lett. 110, 142901 (2017).

25. Xing, J., Guo, E. J., Dong, J., Hao, H., Zheng, Z., \& Zhao, C. High-sensitive switchable photodetector based on $\mathrm{BiFeO}_{3}$ film with in-plane polarization. Appl. Phys. Lett. 106, 033504 (2015).

26. Wang, J., Ma, J., Yang, Y., Chen, M., Zhang, J., Ma, J., \& Nan, C. W. Ferroelectric photodetector with high current on-off ratio $\left(\sim 1 \times 10^{4} \%\right)$ in self-assembled topological nanoislands. ACS Appl. Electron. Mater. 1, 862-868 (2019).

27. Gao, H., Yang, Y., Wang, Y., Chen, L., Wang, J., Yuan, G., \& Liu, J. M. Transparent, flexible, fatigue-free, optical-read, and nonvolatile ferroelectric memories. ACS Appl. Mater. Interfaces 11, 35169-35176 (2019).

28. Huang, W. et al. Highly sensitive switchable heterojunction photodiode based on epitaxial $\mathrm{Bi}_{2} \mathrm{FeCrO}_{6}$ multiferroic thin films. ACS Appl. Mater. Interfaces 10, 12790-12797 (2018).

29. Yan, M. et al. Ferroelectric Synaptic Transistor Network for Associative Memory. Adv. Electron. Mater. 7, 2001276 (2021).

30. Yang, Y. et al. Spin-Filtering Ferroelectric Tunnel Junctions as Multiferroic Synapses for Neuromorphic Computing. ACS Appl. Mater. Interfaces 12, 56300-56309 (2020).

31. $\mathrm{Hu}, \mathrm{H}$. et al. Enhanced resistance switching in ultrathin $\mathrm{Ag} / \mathrm{SrTiO}_{3} /(\mathrm{La}, \mathrm{Sr}) \mathrm{MnO}_{3}$ memristors and their long-term plasticity for neuromorphic computing. Appl. Phys. Lett. 119, 023502 (2021).

32. Li, J., Ge, C., Du, J., Wang, C., Yang, G., \& Jin, K. Reproducible Ultrathin Ferroelectric Domain Switching for High-Performance Neuromorphic Computing. Adv. Mater. 32, 1905764 (2020).

33. Ren, C. et al. Highly Robust Flexible Ferroelectric Field Effect Transistors Operable at High Temperature with Low-Power Consumption. Adv. Funct. Mater. 30, 1906131 (2020). 
34. Ma, C. et al. Sub-nanosecond memristor based on ferroelectric tunnel junction. Nat. Commun. 11, 1-9 (2020).

35. Cheng, S. et al. Highly controllable and silicon-compatible ferroelectric photovoltaic synapses for neuromorphic computing. iScience 23, 101874 (2020).

36. Tan, Z. et al. Thinning ferroelectric films for high-efficiency photovoltaics based on the Schottky barrier effect. NPG Asia Mater. 11, 1-13 (2019).

37. Vrejoiu, I., Le Rhun, G., Pintilie, L., Hesse, D., Alexe, M., \& Gösele, U. Intrinsic ferroelectric properties of strained tetragonal $\mathrm{PbZr}_{0 .}{ }_{2} \mathrm{Ti}_{0 .}{ }_{8} \mathrm{O}_{3}$ obtained on layer-by-layer grown, defect-free single-crystalline films. Adv. Mater. 18, 1657-1661 (2006).

38. Tan, Z. et al. Polarization imprint effects on the photovoltaic effect in $\mathrm{Pb}(\mathrm{Zr}, \mathrm{Ti}) \mathrm{O}_{3}$ thin films. Appl. Phys. Lett. 112, 152905 (2018).

39. Warren, W. L., Dimos, D., Pike, G. E., Tuttle, B. A., Raymond, M. V., Ramesh, R., \& Evans Jr, J. T. Voltage shifts and imprint in ferroelectric capacitors. Appl. Phys. Lett. 67, 866-868 (1995).

40. Pintilie, L., Stancu, V., Vasile, E., \& Pintilie, I. About the complex relation between shortcircuit photocurrent, imprint and polarization in ferroelectric thin films. J. Appl. Phys. 107, 114111 (2010).

41. Pintilie, L., Vrejoiu, I., Hesse, D., LeRhun, G., \& Alexe, M. Ferroelectric polarizationleakage current relation in high quality epitaxial $\mathrm{Pb}(\mathrm{Zr}, \mathrm{Ti}) \mathrm{O}_{3}$ films. Phys. Rev. B 75, 104103 (2007).

42. Lee, D., Baek, S. H., Kim, T. H., Yoon, J. G., Folkman, C. M., Eom, C. B., \& Noh, T. W. Polarity control of carrier injection at ferroelectric/metal interfaces for electrically switchable diode and photovoltaic effects. Phys. Rev. B 84, 125305 (2011).

43. Fang, L., You, L., Zhou, Y., Ren, P., Shiuh Lim, Z., \& Wang, J. Switchable photovoltaic response from polarization modulated interfaces in $\mathrm{BiFeO}_{3}$ thin films. Appl. Phys. Lett. 104, 142903 (2014).

44. Shi, L., Zheng, G., Tian, B., Dkhil, B., \& Duan, C. Research progress on solutions to the sneak path issue in memristor crossbar arrays. Nanoscale Adv. 2, 1811-1827 (2020).

45. Alibart, F., Zamanidoost, E., \& Strukov, D. B. Pattern classification by memristive crossbar circuits using ex situ and in situ training. Nat. Commun. 4, 1-7 (2013).

46. Widrow, B. \& Angel, J. B. Reliable, trainable networks for computing and control. Aerosp. Eng. 21, 78-123 (1962).

47. Yakopcic, C., Alom, M. Z., \& Taha, T. M. Memristor crossbar deep network implementation based on a convolutional neural network. In 2016 International joint 
conference on neural networks (IJCNN) (IEEE, 2016); http://doi.org/10.1109/IJCNN.2016.7727302

48. Fan, H. et al. Large electroresistance and tunable photovoltaic properties of ferroelectric nanoscale capacitors based on ultrathin super-tetragonal $\mathrm{BiFeO}_{3}$ films. J. Mater. Chem. C 5, 3323-3329 (2017).

\section{Acknowledgements}

The authors would like to thank the National Natural Science Foundation of China (Nos. 92163210, U1932125, 52172143, and 62174053), Science and Technology Program of Guangzhou (No. 2019050001), Natural Science Foundation of Guangdong Province (No. 2020A1515010996), and Open Research Projects of Zhejiang Lab (No. 2021MD0AB03).

\section{Author contributions}

Z.F. conceived and supervised the research. B.C., Z.T., G.T., and D.C. prepared the devices. B.C., S.C., B.T., R.T., and X.L. performed the electrical and photovoltaic measurements and relevant results analysis. B.C., W.L., Z.H., M.Z., G.Z., X.G. conducted XRD, AFM, PFM, and TEM measurements and relevant results analysis. Y.C., S.D., and M.Q. carried out simulation works. B.C., Z.F., X.G., and J.-M.L. wrote and revised the manuscript.

\section{Additional information}

Supplementary Information accompanies this paper at [to be inserted].

Competing interests: The authors declare no competing interests.

Reprints and permission information is available online at http://npg.nature.com/reprintsandpermissions/

Publisher's note: Springer Nature remains neutral with regard to jurisdictional claims in published maps and institutional affiliations.

\section{Figure Captions}

Fig. 1 Hardware implementations of machine vision. Schematics for (a) conventional Von Neumann system and (b) emerging neuromorphic visual system. (c) Near- and (d) in-sensor computing architectures for the neuromorphic visual system. The proposed FE-PS-NET uses the architecture shown in d, where FE-PSs act as reconfigurable photosensors.

Fig. 2 Polarization switching behavior and polarization-tuned multilevel nonvolatile photovoltaic responses in FE-PS. (a) Schematics illustrating the device structure of the Pt/ 
PZT (epitaxial film)/SRO FE-PS (left panel) and the crystal structure of PZT (right panel). (b) AFM topography image and (c) high-resolution cross-sectional TEM image of the epitaxial PZT film. (d) Bipolar, (e) positive monopolar, and (f) negative monopolar $P$ - $V$ hysteresis loops measured with different pulse voltages. Illuminated $I-V$ characteristics measured after applying different $(\mathrm{g})$ positive pulses and $(\mathrm{h})$ negative pulses to the FE-PS initiated by $-3 \mathrm{~V}$ and $+3 \mathrm{~V}$ pulses, respectively. In e-h, the different polarizations states are schematically illustrated by the configurations of the four black solid arrows. (i) $I_{\mathrm{sc}}$ and $V_{\mathrm{oc}}$ as a function of the write pulse voltage $\left(V_{\mathrm{p}}\right)$.

Fig. 3 Synaptic behavior and operation mechanism of FE-PS. (a) One-cycle LTD and LTP characteristics in which the photoresponsivity represents the weight. Inset shows the schematics of the applied pulse trains. (b) Multi-cycle LTD and LTP characteristics. (c) Schematic energy band diagrams of the FE-PS in the complete $P_{\text {up }}$ (left panel) and $P_{\text {down }}$ (right panel) states. The polarization switching can tune the Schottky barrier heights and built-in fields at the two interfaces, thus modifying the magnitude and direction of the overall output photocurrent.

Fig. 4 In-sensor MAC operations in FE-PS-NET. (a) Schematic illustration of the architecture of the FE-PS-NET. (b) Schematic circuit diagram for a pixel in the FE-PS-NET. (c) Schematic circuit diagram for a $1 \times 2$ FE-PS-NET. (d,e) Time-resolved currents $\left(I_{1}\right)$ measured during the applications of different illuminations to the $(1,1)$ and $(1,2)$ FE-PSs. In $d$ the $(1,1)$ and $(1,2)$ FE-PSs are both set in the complete $P$ up state, while in e the two devices are set in the complete $P_{\text {down }}$ and $P_{\text {up }}$ states, respectively. In each period during which at least one FE-PS is illuminated, the MAC operation is directly expressed as the equation containing the experimental values of photoresponsivity, optical power, and output current. Their corresponding units are $\mathrm{mA} / \mathrm{W}, \mu \mathrm{W}$, and $\mathrm{nA}$, respectively (not shown). (f) Schematic circuit diagram for a $2 \times 2$ FE-PS-NET. (g) Time-resolved currents $\left(I_{1}\right)$ measured during the applications of different illuminations to the $(1,1),(1,2),(2,1)$ and $(2,2)$ FE-PSs. All the FEPSs are set in the complete $P_{\text {up }}$ state, and the illumination sequences are indicated in the corresponding periods while the optical power for illumination $(\sim 25 \mu \mathrm{W})$ is not shown.

Fig. 5 Implementation of pattern classification. (a) Two sets of patterns representing letters ' $\mathrm{X}$ ' and ' $\mathrm{T}$ '. (b) Schematic diagrams showing the operation and the circuit structure of a $1 \times 9$ FE-PS-NET. The 9 FE-PSs are illuminated following the signals translated from the input pattern. (c) From left to right: calculated dimensionless weights, theoretical photoresonsivities scaled from dimensionless weights, actual photoresonsivities right after programming, actual photoresonsivities after pattern classification. The unit of photoresponsivity is $\mathrm{mA} / \mathrm{W}$ (not 
shown). (d) Output currents ( $\left.I_{1}\right)$ during the presentations of different input patterns. The theoretical $I_{1}$ values are indicated by the "star" symbols. The unit of $I_{1}$ is nA (not shown) (e) Neuronal outputs obtained by feeding the output currents to the sigmoid function.

Fig. 6 Implementation of edge detection. (a) Schematic illustration of the operations for the edge detection. The convolution is implemented by sequentially presenting the sub-images whose pixel values are translated to the illumination signals to the kernels based on FE-PSNET. The merging, normalization, and binarization of output currents are performed in software. (b) From left to right: theoretical dimensionless weights, theoretical photoresonsivities scaled from dimensionless weights, actual weights right after programming, and actual photoresonsivities after edge detection, for Kernel 1 and 2. The unit of photoresponsivity is mA/W (not shown). (c) Output currents $I_{1}$ (left panel) and $I_{2}$ (right panel) after the convolution with the two kernels. The values outside the brackets are the actual $I_{1}$ and $I_{2}$ values while those in the brackets are the differences between the theoretical $I_{1}$ and $I_{2}$ values and their corresponding actual values. The units of $I_{1}$ and $I_{2}$ are nA (not shown) (d) Image obtained by merging $I_{1}$ and $I_{2}$ (left panel) and final output image after normalization and binarization showing the detected edge (right panel). 
a

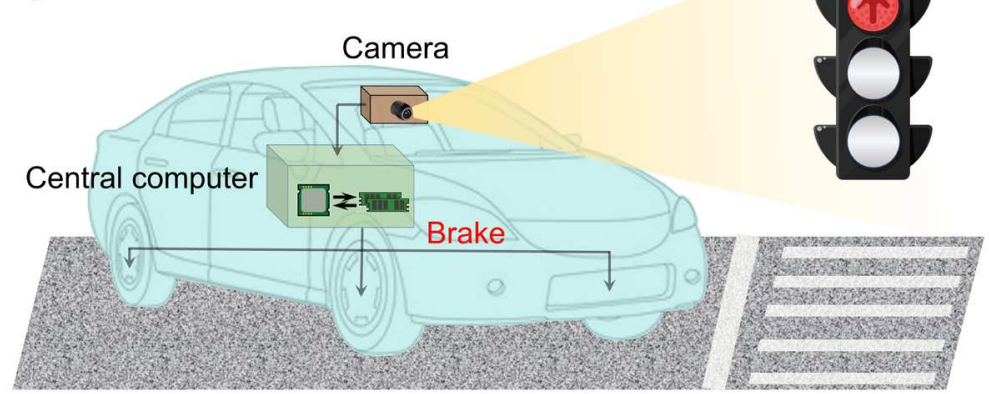

b

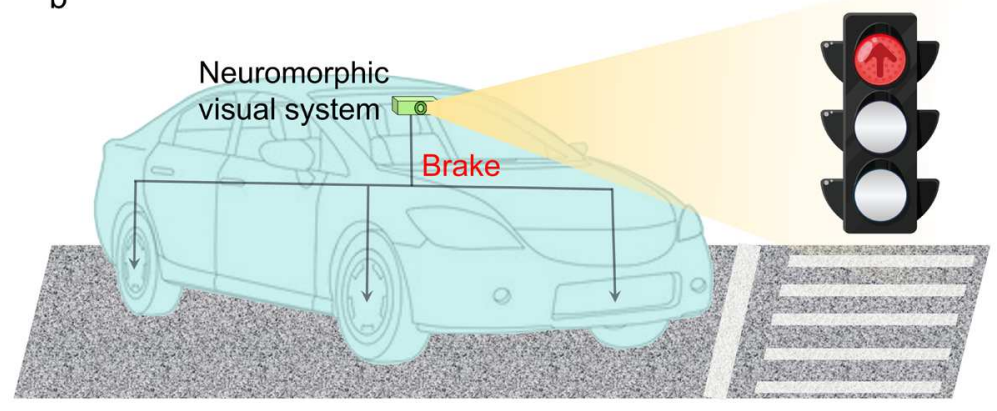

C

\section{Near-sensor computing architecture}

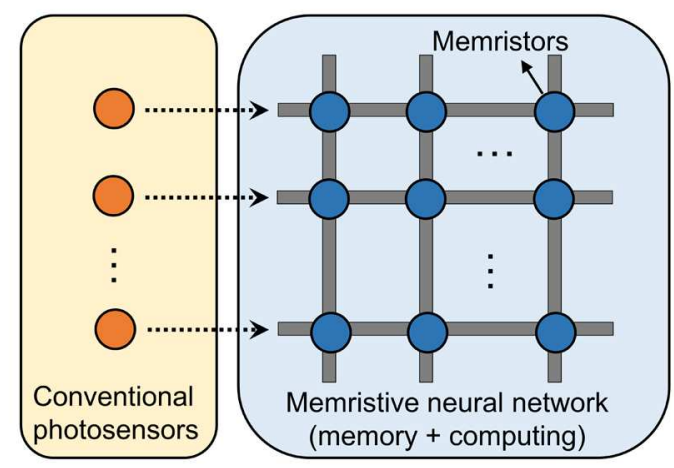

In-sensor computing architecture

Reconfigurable photosensors

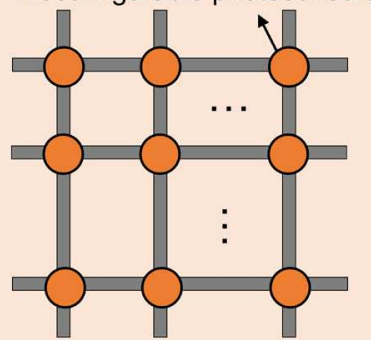

Sensory neural network (sensing + memory + computing)

Figure 1 
a

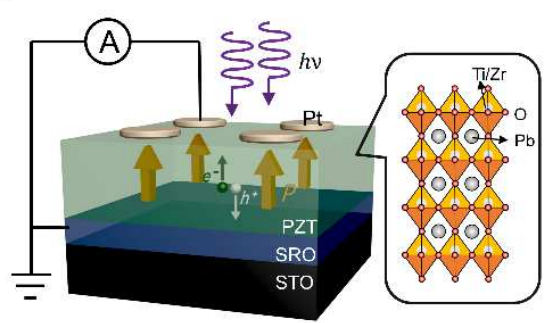

b

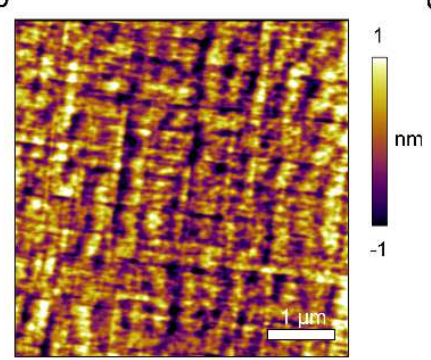

c

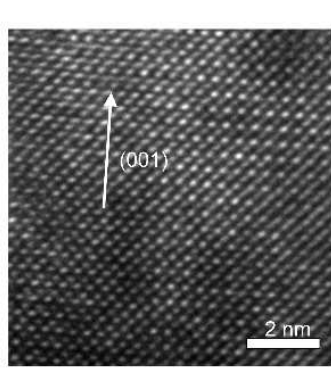

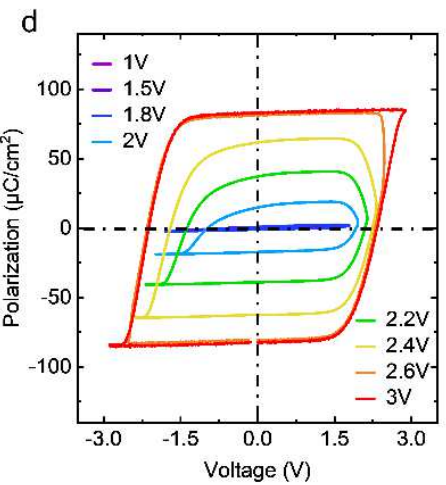
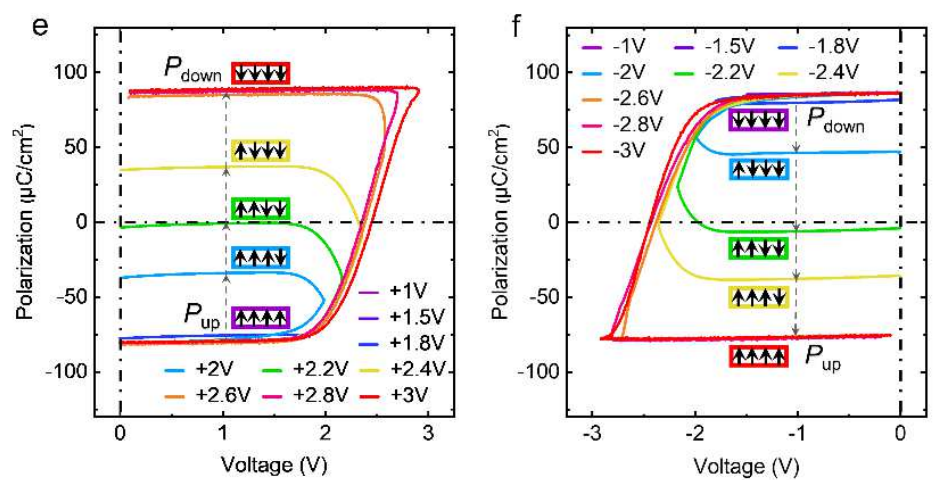

g

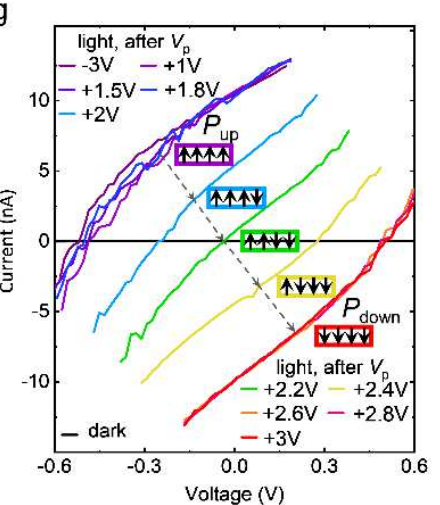

h
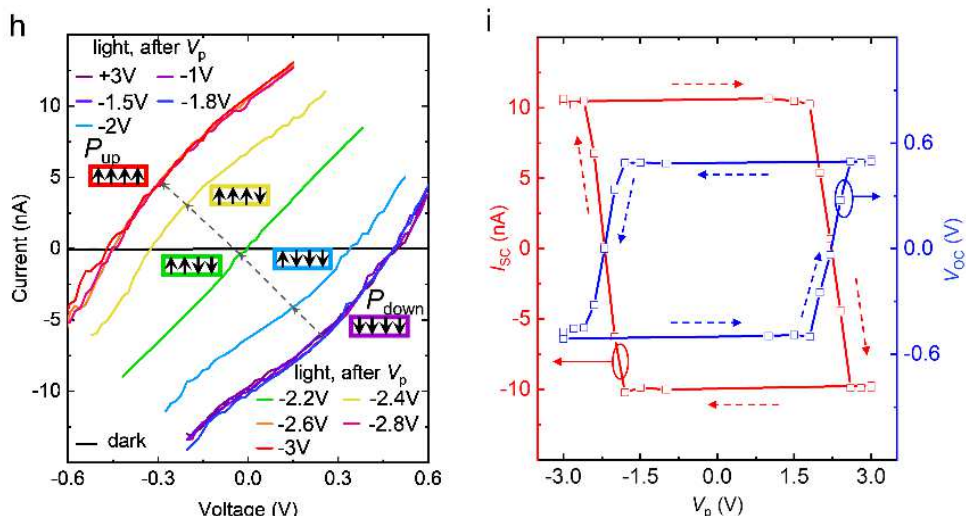

Figure 2 
a

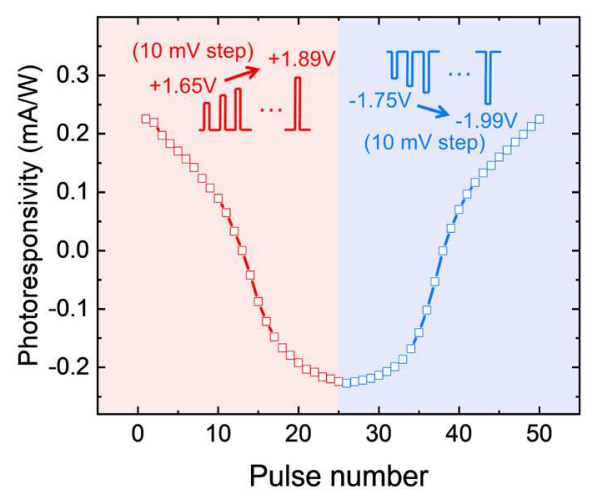

C

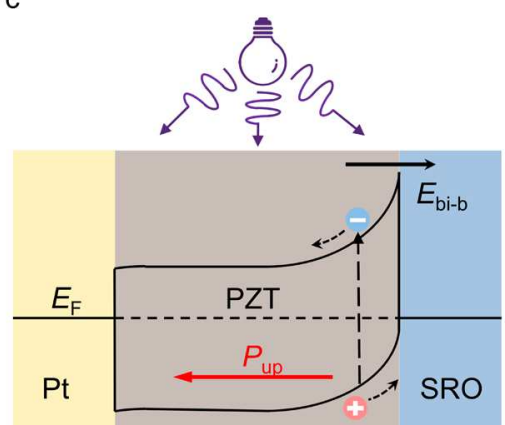

b
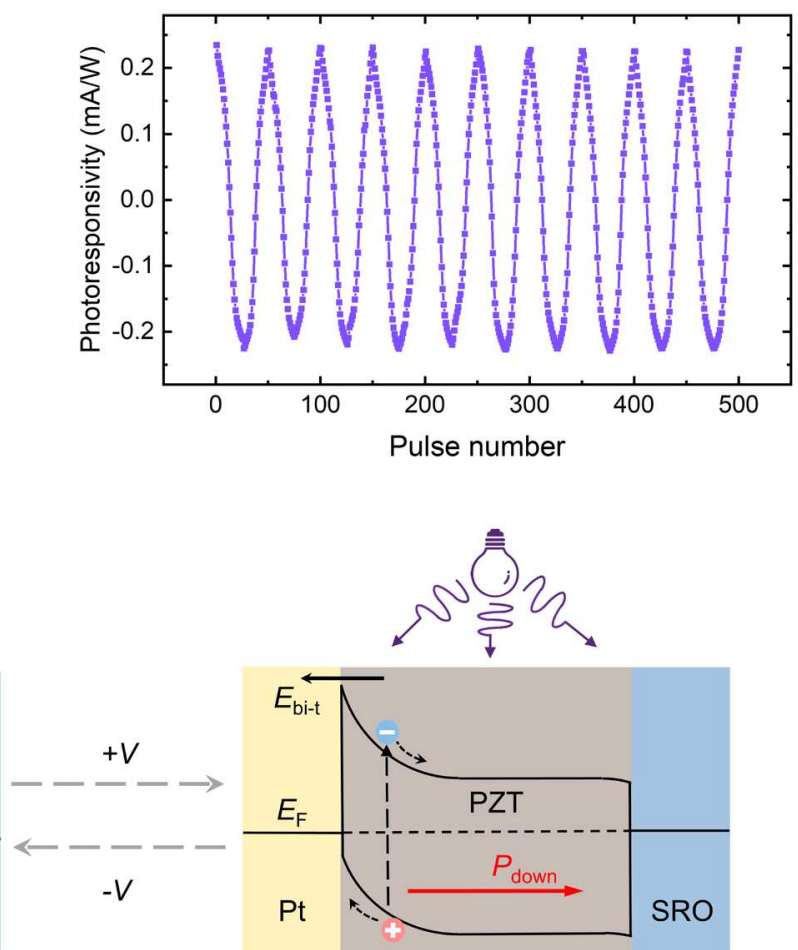

Figure 3 

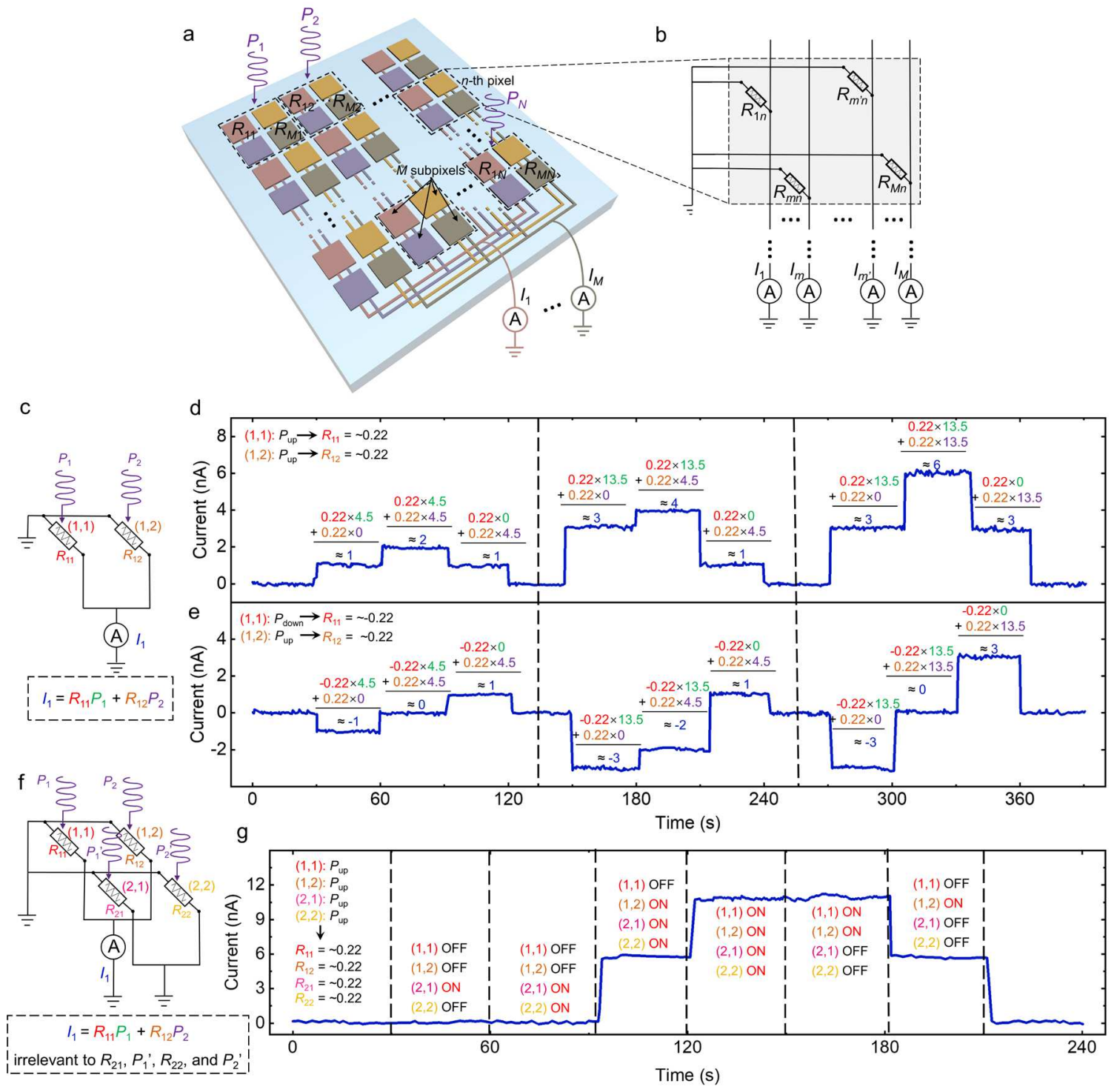

Figure 4 
Pattern 'X', class 1

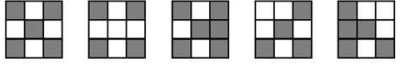

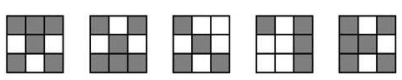

国㽢田

Pattern ' $T$ ', class 0

国困国国田

四四四田果

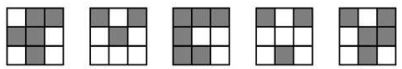

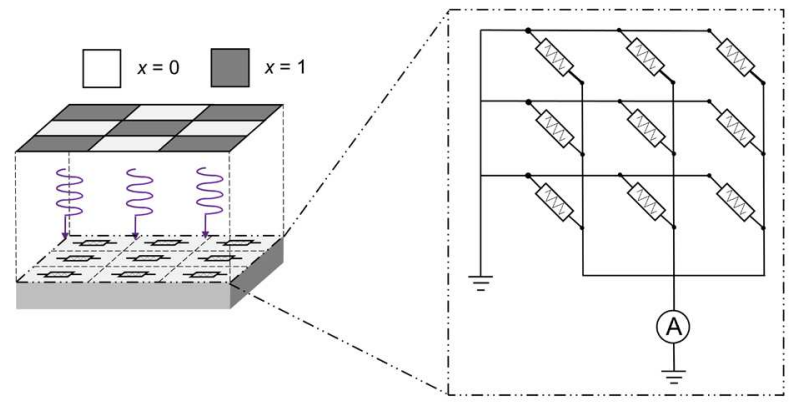

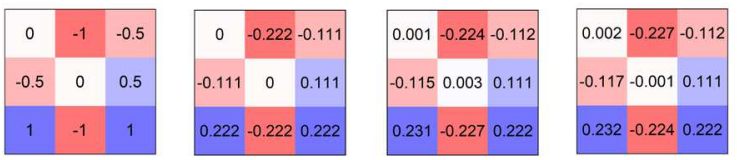

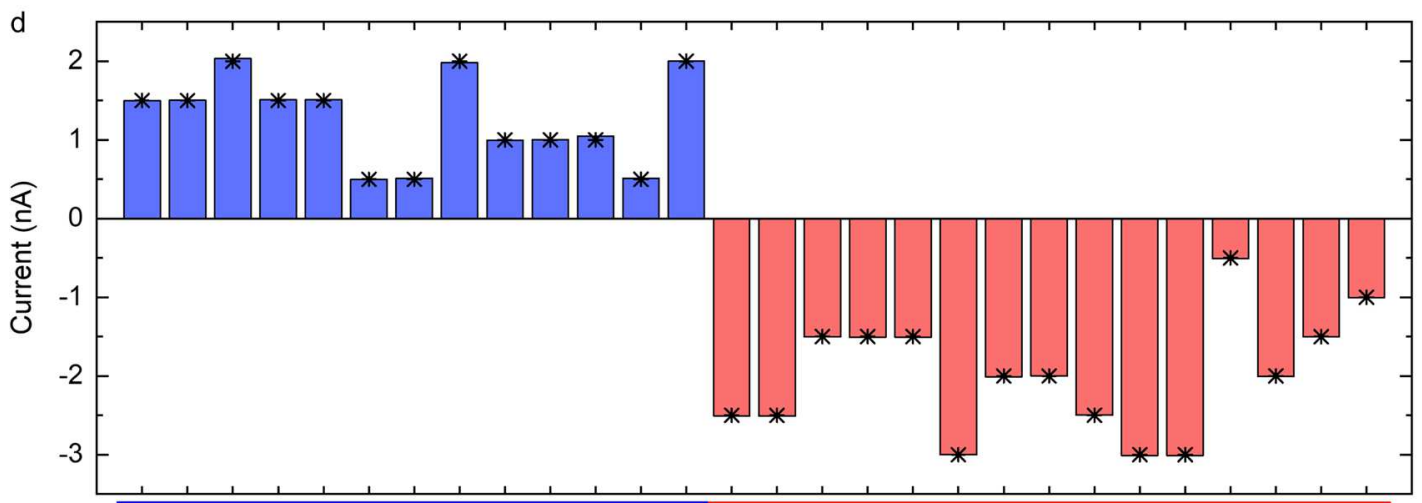

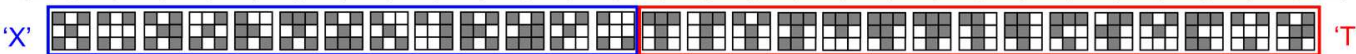

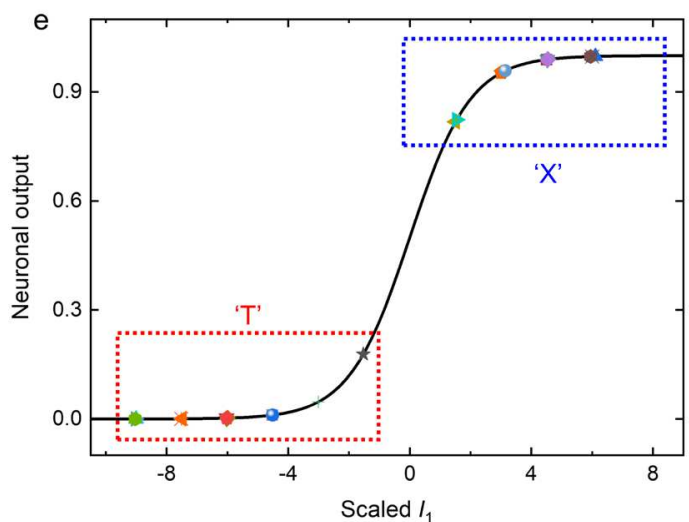

Figure 5 
a

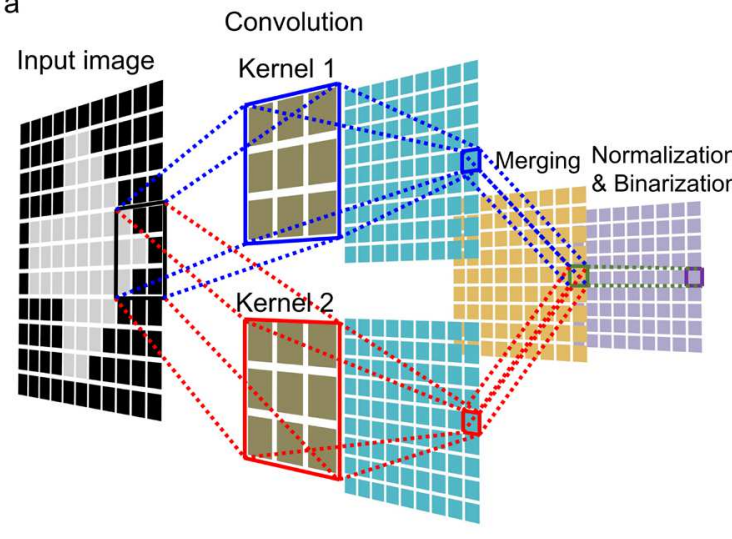

b

Kernel 1

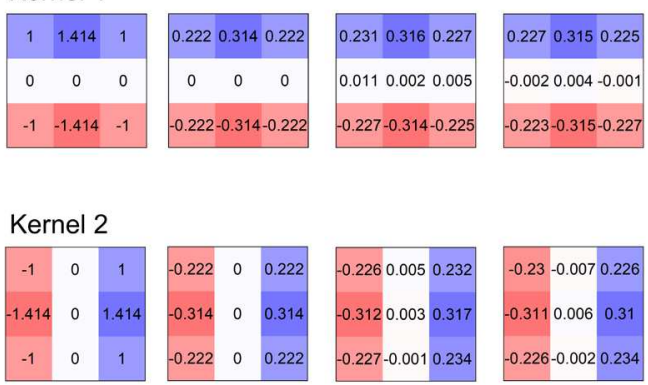

\section{$I_{2}$}

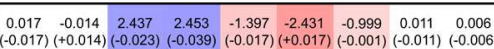

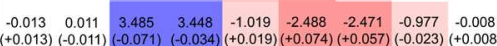

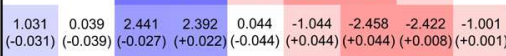

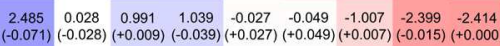

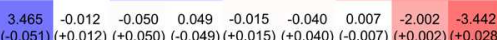

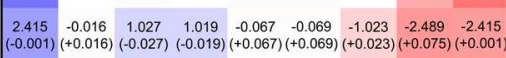

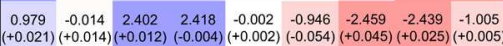

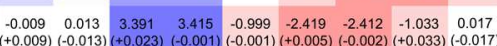
(-0.009) $(-0.013)(+0.023)$ (3.

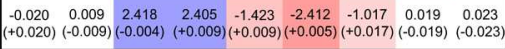

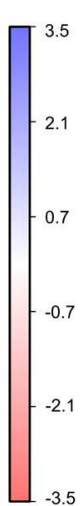

d

$\left|I_{1}\right|+\left|I_{2}\right|$

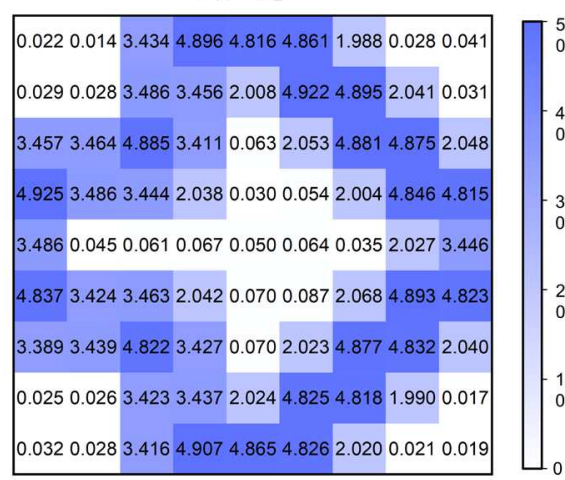

Figure 6 


\section{Supplementary Files}

This is a list of supplementary files associated with this preprint. Click to download.

- Sl.docx 